Charging/discharging dynamics of CdS and CdSe films under photoillumination using dynamic $x$-ray photoelectron spectroscopy

Hikmet Sezen, and Sefik Suzer

Citation: Journal of Vacuum Science \& Technology A: Vacuum, Surfaces, and Films 28, 639 (2010); doi: 10.1116/1.3289319

View online: http://dx.doi.org/10.1116/1.3289319

View Table of Contents: http://avs.scitation.org/toc/jva/28/4

Published by the American Vacuum Society

Contact Hiden Analytical for further details: w www.HidenAnalytical.com E info@hiden.co.uk CLICK TO VIEW our product catalogue

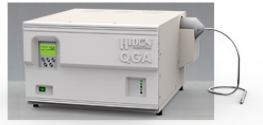

Gas Analysis

dynamic measurement of reaction gas streams catalysis and thermal analysis molecular beam studies dissolved species probes fermentation, environmental and ecological studies

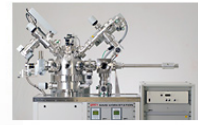

Surface Science UHVTPD , SIMS

, end point detection in ion beam etch elemental imaging - surface mappin

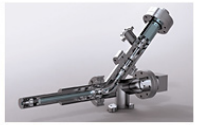

Plasma Diagnostics plasma source characterization etch and deposition process reaction kinetic studies analysis of neutral and radical species

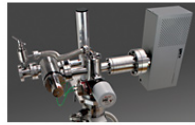

Vacuum Analysis ' partial pressure measurement and control of process gases , reactive sputter process control , vacuum diagnostics - vacuum coating process monitoring 


\title{
Charging/discharging dynamics of CdS and CdSe films under photoillumination using dynamic x-ray photoelectron spectroscopy
}

\author{
Hikmet Sezen and Sefik Suzer ${ }^{\mathrm{a})}$ \\ Department of Chemistry, Bilkent University, 06800 Ankara, Turkey
}

(Received 15 October 2009; accepted 14 December 2009; published 29 June 2010)

\begin{abstract}
Thin films of $\mathrm{CdS}$ and $\mathrm{CdSe}$ are deposited on $\mathrm{HF}$-cleaned $\mathrm{SiO}_{2} / \mathrm{Si}$ substrates containing $\sim 5 \mathrm{~nm}$ thermally grown silicon oxide. x-ray photoelectron spectroscopy (XPS) data of these films are collected in a dynamic mode, which is based on recording the spectrum under modulation with an electrical signal in the form of $\pm 10 \mathrm{~V}$ square-wave pulses. Accordingly, all peaks are twined and shifted with respect to the grounded spectrum. The binding energy difference between the twinned peaks of a dielectric system has a strong dependence on the frequency of the electrical stimuli. Therefore, dynamic XPS provides a means to extract additional properties of dielectric materials, such as effective resistance and capacitance. In this work, the authors report a new advancement to the previous method, where they now probe a photodynamic process. For this reason, photoillumination is introduced as an additional form of stimulus and used to investigate the combined optical and electrical response of the photoconductive thin films of CdS and CdSe using dynamic XPS. (C) 2010 American Vacuum Society. [DOI: 10.1116/1.3289319]
\end{abstract}

\section{INTRODUCTION}

Photoconductive materials have gained renewed interest in recent years due to the advancements in controlling their electronic and optical properties, which exhibit strong size, shape, and chemical composition dependence. ${ }^{1,2}$ Photoconductivity is basically described as a conductivity enhancement or resistivity diminishment under photoillumination, and CdS and CdSe are well known and widely used photoconductive materials. ${ }^{3}$ x-ray photoelectron spectroscopy (XPS) has been used for extracting electrical information from poorly conducting systems or domains. ${ }^{4-10}$ Furthermore, the use of XPS for probing photovoltaic effects in such systems has recently been reported by Cohen and coworkers, where they perform the so-called chemically resolved electrical measurements under controlled electrical conditions. ${ }^{11-15}$ Our measurements are similar in concept but differ drastically in implementation since we also introduce time-dependent measurements, either by recording directly the time-dependent signals with millisecond resolution or harvesting information from the frequency dependence of the response of the system to the external electrical stimuli. We have named the latter technique as dynamical XPS, which is basically implemented by recording XPS data while applying square-wave (or in other waveforms) electrical pulses with different frequencies to the sample. ${ }^{16-22}$ In this work, we introduce another form of stimuli, the photoillumination, and investigate $\mathrm{CdS}$ and $\mathrm{CdSe}$ thin films using dynamical XPS under optical excitation. Although common photovoltage effect has been detected and reported using XPS, ${ }^{23-25}$ to our knowledge, this is the first time that the dynamics of the process has ever been reported.

a) Author to whom correspondence should be addressed; electronic mail: suzer@fen.bilkent.edu.tr

\section{EXPERIMENT}

$\mathrm{CdS}$ and CdSe thin films are cast from their slurry acetone solutions onto $\mathrm{Si}$ substrates containing $\sim 5 \mathrm{~nm}$ thermally grown oxide layer. A Kratos ES300 photoelectron spectrometer with nonmonochromatic $\mathrm{Mg} K \alpha$ x rays (at $1253.6 \mathrm{eV}$ ) source is employed for recording XPS measurements. The pressure in the analysis chamber is kept at around $10^{-8}$ Torr during data recording. The $\mathrm{x}$ rays are directed to the sample with a $45^{\circ}$ angle and the electron analyzer is located at the top of the sample with a $90^{\circ}$ orientation with respect to the surface of the sample plane, so the take-off angle is $90^{\circ}$. The sample rod is externally connected to either the ground, to a dc power supply, or to the pulse generator from both top and bottom sides of the sample, as illustrated in Fig. 1. One more power supply is used to supply a $-3 \mathrm{~V}$ potential to the filament to accelerate the emitted low-energy electrons from the hot filament. This filament is used as the source of lowenergy electrons to neutralize or negatively charge the sample. Stanford Research System DS340 is used as the pulse generator. A continuous wave $180 \mathrm{~mW}$ solid state laser (CrystaLaser) at $405 \mathrm{~nm}$ is used for photoillumination to implement the photodynamic processes, as also shown in Fig. 1. XPS peaks are curve fitted by a third-party free program, XPSPEAK95 version 2.0. ${ }^{26}$ The binding energy scale of XPS is calibrated according to the peak positions of Au $4 f$ $(84.0 \mathrm{eV})$ and $\mathrm{Ag} 3 d(368.3 \mathrm{eV})$. A MATLAB ${ }^{\circledR}$ routine is used to calculate the dynamical XPS spectra, modulated at any form and frequency, under electrical stimuli. This program also plots the calculated binding energy difference between the twinned peaks against the value of the corresponding pulse frequency for any arbitrarily assigned resistance $(R)$ and capacitance $(C)$ values. The solution is obtained by trying a set of arbitrarily chosen $R$ and $C$ values in order to find a good match between the calculated and the experimentally measured binding energy differences versus the applied fre- 


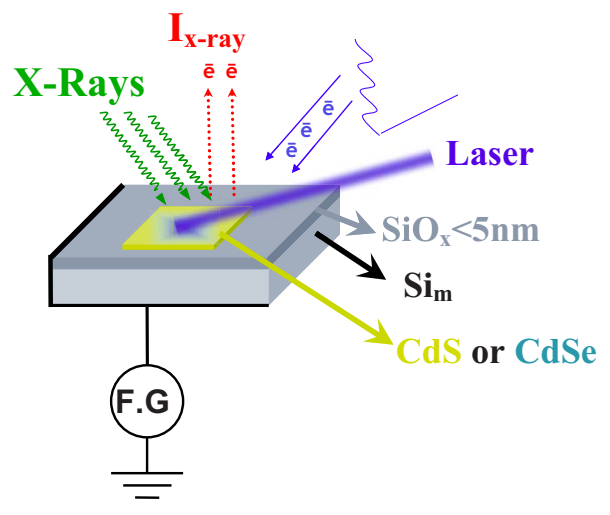

FIG. 1. (Color online) Schematic representation of the photoilluminated dynamic XPS measurements.

quency of the electrical stimuli. Detailed information about the experimental method and software is available from our previously published articles. ${ }^{18-22}$

\section{RESULTS AND DISCUSSION}

The basic phenomenon responsible for our measurements is the charging of dielectric materials or domains due to the photoemission process. This charging, or surface charging, has been an important experimental obstacle and tremendous effort has been directed to minimize it. However, as was also mentioned in the Introduction section, several published reports show that this surface charging can also be utilized to get additional chemical, electrical, and physical information. ${ }^{4-10,13,18-20}$ Along these directions, we have recently developed a technique for recording the shifts in the binding energy positions of the XPS peaks in response to different forms of electrical stimuli for probing dynamics of charging/discharging processes in thin dielectric films, which we have named as dynamical XPS and is schematically represented in Fig. 1. ${ }^{18-22}$ In this technique, dielectric layers are modeled as an electrical unit of parallel connected resistor and capacitor pairs, as illustrated in Fig. 2. In this circuit, while part of a typical resistor, $R$, and capacitor, $C$, couple represents the thermally grown $\mathrm{SiO}_{2}$ layer, a photoresistor, $R_{\mathrm{ph}}$, and another capacitor, $C_{\mathrm{ph}}$, couple represents the photo-

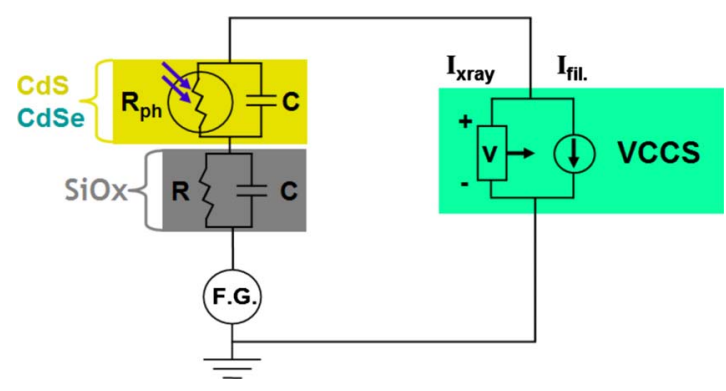

FIG. 2. (Color online) Circuit used for the simulation of the dynamics of charging/discharging behavior of the dielectric film(s). The $\mathrm{SiO}_{2}$ dielectric layer is approximated by a capacitor $(C)$ and a resistor $(R)$, and another parallel connected photoresistor, $R_{\mathrm{ph}}$ and $C_{\mathrm{ph}}$, unit represents the photoconductive CdSe and/or CdS layer(s). VCCS is the voltage controlled current source.

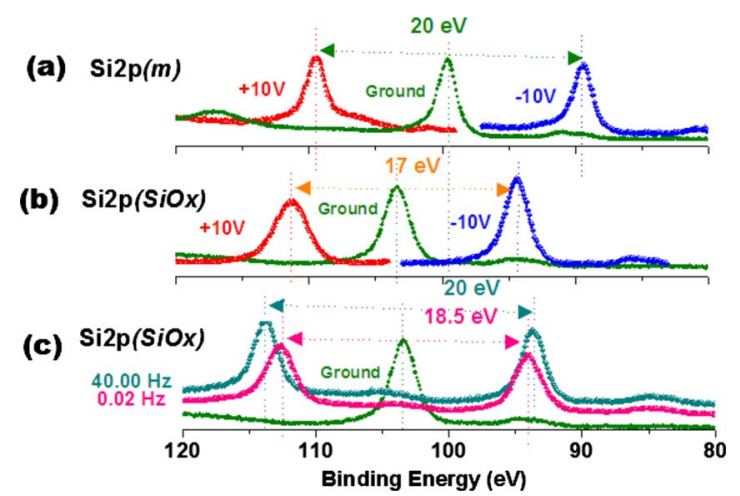

FIG. 3. (Color online) XPS spectra of the Si $2 p$ region of the Si samples which are (a) HF-cleaned and [(b) and (c)] containing $\sim 100 \mathrm{~nm}$ thermally grown silicon oxide layer. Olive-colored spectra belong to when the samples are connected to ground, and red and blue-colored spectra belong when samples are subjected to +10 and $-10 \mathrm{~V}$ dc bias, respectively. Cyan and magenta-colored spectra belong to the $\mathrm{SiO}_{2}$ sample when it is subjected 40 and $0.02 \mathrm{~Hz}$ square-wave pulses.

conductive CdS or CdSe thin films. $I_{\mathrm{x} \text { ray }}$ and $I_{\text {fil }}$ are the two main current sources, which correspond to the photoemitted electrons and the low-energy electrons from the hot filament, respectively. They are incorporated in this lumped electrical circuit as a voltage controlled current source (VCCS) unit. If the response of the circuit is calculated, the time variant voltage on the pairs of resistor and capacitor unit provides the dynamical behavior of charging/discharging of dielectric films for any form and frequency of the electrical stimuli. ${ }^{18}$

We now present the photoillumination as an additional form of stimuli and investigate the combined optical and electrical response of the CdS and CdSe thin films. In Fig. $3(\mathrm{a})$, the XPS spectrum of the $\mathrm{Si} 2 p$ region of a pure HFcleaned $\mathrm{Si}$ wafer is shown when the sample is under both positive and negative $10 \mathrm{~V}$ dc bias. Since Si has enough conductivity, the position of the $\mathrm{Si} 2 p$ peak exhibits only the trivial shifts of +10.0 and $-10 \mathrm{eV}$ with respect to its grounded position. However, for another Si sample containing $\sim 100 \mathrm{~nm}$ thermally grown silicon oxide, the corresponding oxide Si $2 p$ peak exhibits smaller than $10.0 \mathrm{eV}$ shifts due to charging, as shown in Fig. 3(b). Hence, a measurement of less than $20.0 \mathrm{eV}$ binding energy difference is an experimental evidence of charge accumulation on the surface of the dielectric layer under investigation $\left(\mathrm{SiO}_{2}\right.$ in this case).

Upon application of the square-wave excitation, all XPS peaks are twined due to the application of positive and negative potentials successively several times in the same recording. Since a time-dependent stimulation is operative, the measured binding energy difference between the twinned peaks of the dielectric systems exhibit strong frequency dependence as well. At low frequencies, since the sample has enough time to charge and/or discharge, a smaller than $20.0 \mathrm{eV}$ is recorded; on the other hand, the binding energy difference between the twinned $\mathrm{Si} 2 p$ peaks of the $\mathrm{SiO}_{2}$ layer recovers at higher frequencies because the sample does not have enough time to charge and/or discharge, as displayed in Fig. 3(c). 


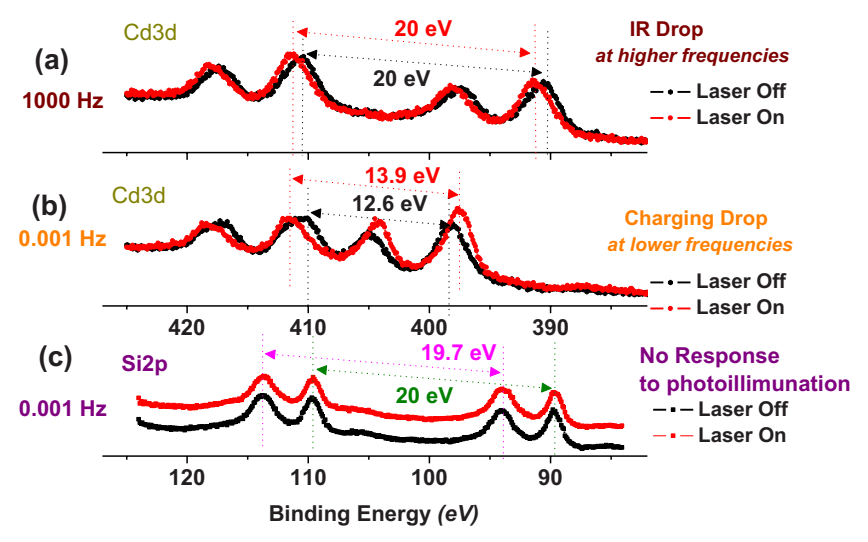

FIG. 4. (Color online) Dynamic XPS spectra of the thin film of CdSe on $\mathrm{SiO}_{2}$ under square-wave excitation and with (red lines) and without photoillumination (black lines) of the $\mathrm{Cd} 3 d$ region at (a) $1000 \mathrm{~Hz}$ and (b) $0.001 \mathrm{~Hz}$ and (c) Si $2 p$ region at $0.001 \mathrm{~Hz}$.

While applying the square-wave excitation in the $10^{-3}-10^{3} \mathrm{~Hz}$ range to the photoconductive thin films of $\mathrm{CdS}$ and $\mathrm{CdSe}$, we record the spectra twice at the laser-off and laser-on modes. As shown in Fig. 4(b), the binding energy difference, between the twinned $\mathrm{Cd} 3 d$ spin-orbit doublet peaks of the CdSe layer, under $0.001 \mathrm{~Hz}$ square-wave excitation and laser-off mode, is measured as $12.6 \mathrm{eV}$, corresponding to a severely charged sample. When the laser illumination is switched on, and at this same lower frequency, the binding energy difference increases to $13.9 \mathrm{eV}$ due to a decrease in the effective $R_{\mathrm{ph}}$ of CdSe. At high frequencies, the system's response is recovered, and a $20.0 \mathrm{eV}$ difference is recorded, as shown in Fig. 4(a), but this time there is an offset in the positions between the laser-on and laser-off data. As was thoroughly discussed in our previous paper, this offset is also related to a decrease in the effective $R_{\mathrm{ph}}$ upon illumination through the voltage drop produced as a result of the average current passing from the sample, which we name it as $I_{\mathrm{tot}} \cdot R_{\mathrm{ph}}$ drop. $^{22}$ The Si $2 p$ region is also recorded to ensure that photoconductive behavior is specific only to the thin films of CdS or CdSe. In addition, as shown in Fig. 4(c) the $\mathrm{Si} 2 p$ peak of the $\mathrm{SiO}_{2}$ layer displays a charging shift but the photoillumination does not produce any measurable change in this shift, due, most probably, to the fast recombination processes of the photoexcited electron-hole pairs. ${ }^{24,27}$ Hence, neither the $\mathrm{Si}$ nor the $\mathrm{SiO}_{2}$ layers respond to photoillumination, but the dielectric $\mathrm{SiO}_{2}$ layer exhibits slight charging shifts, as evidenced by its measured binding energy difference of $19.7 \mathrm{eV}$ at $0.001 \mathrm{~Hz}$, while the underlying $\mathrm{Si}$ layer exhibits exactly $20.0 \mathrm{eV}$ difference.

In order to get a more detailed electrical information, a full set of frequency dependence data is collected in the range of $10^{-3}-10^{3} \mathrm{~Hz}$, which are shown in Fig. 5, as plots of the binding energy differences between twinned $\mathrm{Cd} 3 d$ peaks of the CdSe layer under photoillumination and without photoillumination versus the logarithm of the frequency. The effective values of photoresistance and capacitance are obtained by fitting the data. As a result, $\sim 7.5 \mathrm{M} \Omega$ resistance and $40 \mathrm{nF}$ capacitance are obtained in the laser-off mode for

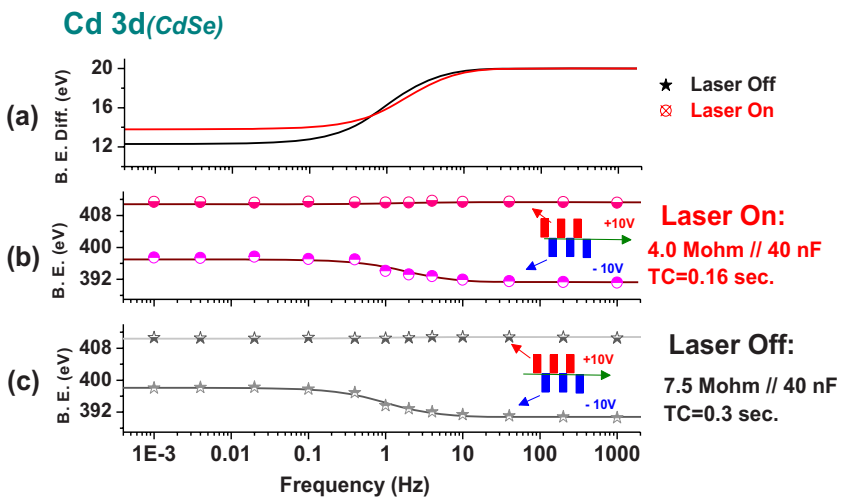

FIG. 5. (Color online) Measured binding energies (b) and the binding energy differences (a) between the twinned Cd $3 d$ peaks of the CdSe layer together with the calculated data with and without photoillumination. (c) The points represent the experimental data, and the solid lines represent the results of the calculation for the values of $R$ and $C$ indicated.

the CdSe layer. As expected, a smaller value of $\sim 4.0 \mathrm{M} \Omega$ resistance and the same value of $40 \mathrm{nF}$ capacitance in the laser-on mode are calculated. It is clear that while the effective capacitance value of the photoconductive CdSe layer is constant, the effective $R_{\mathrm{ph}}$ changes significantly upon photoillumination.

The same experimental data are collected and a similar calculation is conducted for the photoconductive thin film of CdS, as shown in Fig. 6. As a result, $6.5 \mathrm{M} \Omega$ resistance and $20 \mathrm{nF}$ capacitance values are calculated for the laser-off mode and $\sim 4.0 \mathrm{M} \Omega$ resistance and $20 \mathrm{nF}$ capacitance values are obtained for the laser-on mode. Similar to the CdSe case, when the sample is excited with photoillumination, the effective resistance value has a significant decrease, but the value of capacitance remains unchanged.

\section{CONCLUSIONS}

In summary, by subjecting samples to square-wave excitation at a range of $10^{3}-10^{-3} \mathrm{~Hz}$, while recording XPS data, the electrical information with chemical specificity is extracted by the dynamic XPS technique. Moreover, photocon-

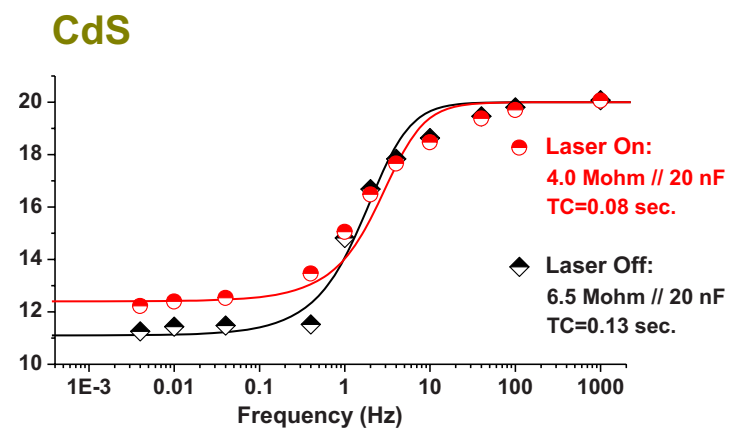

FIG. 6. (Color online) Binding energy differences between twinned Cd $3 d$ peaks of the CdS layer under photoillumination (red) and without photoillumination (black) at different frequencies. The points represent the experimental data, and the solid lines represent the calculated results for the values of $R$ and $C$ indicated. 
ductive behaviors of the $\mathrm{CdS}$ and CdSe thin films are assessed by recording similar data with and without photoillumination.

\section{ACKNOWLEDGMENTS}

This work was partially supported by TUBA (Turkish Academy of Sciences), TUBITAK (The Scientific and Technological Research Council of Turkey) through Grant No. 106T409, and by the European Union 7th Framework Project Unam-Regpot (Grant No. 203953).

${ }^{1}$ X. Peng, L. Manna, W. Yang, J. Wickham, E. Scher, A. Kadavanich, and A. P. Alivisatos, Nature (London) 404, 59 (2000).

${ }^{2}$ Z. A. Peng and X. G. Peng, J. Am. Chem. Soc. 123, 1389 (2001).

${ }^{3}$ D. P. Amalnerkar, Mater. Chem. Phys. 60, 1 (1999).

${ }^{4}$ W. M. Lau, J. Appl. Phys. 67, 1504 (1990).

${ }^{5}$ J. Cazaux, J. Electron Spectrosc. Relat. Phenom. 105, 155 (1999).

${ }^{6}$ K. Shabtai, I. Rubinstein, S. R. Cohen, and H. Cohen, J. Am. Chem. Soc. 122, 4959 (2000).

${ }^{7}$ S. Suzer, Anal. Chem. 75, 7026 (2003).

${ }^{8}$ G. Ertas and S. Suzer, Surf. Interface Anal. 36, 619 (2004)

${ }^{9}$ M. Dubey, I. Gouzman, S. L. Bernasek, and J. Schwartz, Langmuir 22, 4649 (2006).

${ }^{10}$ I. Gouzman, M. Dubey, M. D. Carolus, J. Schwartz, and S. L. Bernasek, Surf. Sci. 600, 773 (2006).
${ }^{11}$ R. Buller, H. Cohen, E. Minkin, R. Popovitz-Biro, E. Lifshitz, and M. Lahav, Adv. Funct. Mater. 12, 713 (2002).

${ }^{12}$ A. Samokhvalov, R. W. Gurney, M. Lahav, S. Cohen, H. Cohen, and R. Naaman, J. Phys. Chem. B 107, 4245 (2003).

${ }^{13}$ H. Cohen, R. Maoz, and J. Sagiv, Nano Lett. 6, 2462 (2006).

${ }^{14}$ H. Cohen, S. K. Sarkar, and G. Hodes, J. Phys. Chem. B 110, 25508 (2006).

${ }^{15}$ S. K. Sarkar, G. Hodes, L. Kronik, and H. Cohen, J. Phys. Chem. C 112 , 6564 (2008).

${ }^{16}$ U. K. Demirok, G. Ertas, and S. Suzer, J. Phys. Chem. B 108, 5179 (2004).

${ }^{17}$ G. Ertas, U. K. Demirok, A. Atalar, and S. Suzer, Appl. Phys. Lett. 86, 183110 (2005).

${ }^{18}$ S. Suzer and A. Dana, J. Phys. Chem. B 110, 19112 (2006).

${ }^{19}$ H. Sezen, G. Ertas, A. Dana, and S. Suzer, Macromolecules 40, 4109 (2007).

${ }^{20}$ S. Suzer, H. Sezen, and A. Dana, Anal. Chem. 80, 3931 (2008).

${ }^{21}$ S. Suzer, H. Sezen, G. Ertas, and A. Dâna, J. Electron Spectrosc. Relat. Phenom. 176, 52 (2010).

${ }^{22}$ H. Sezen, G. Ertas, and S. Suzer, J. Electron Spectrosc. Relat. Phenom. (in press).

${ }^{23}$ N. R. Huck, R. S. C. Smart, and S. M. Thurgate, Surf. Sci. 169, L245 (1986).

${ }^{24}$ N. J. Halas and J. Bokor, Phys. Rev. Lett. 62, 1679 (1989).

${ }^{25}$ W. Jaegermann, C. Pettenkofer, and B. A. Parkinson, Phys. Rev. B 42, 7487 (1990).

${ }^{26}$ R. W. M. Kwok, available at http://phy.cuhk.edu.hk/ surface/XPSPEAK/.

${ }^{27}$ W. Widdra et al., Surf. Sci. 543, 87 (2003) 